\title{
ARTICLE OPEN \\ In-plane orientation-dependent metal-insulator transition in vanadium dioxide induced by sublattice strain engineering
}

\author{
Weizheng Liang ${ }^{1}$, Zengqing Zhuo ${ }^{2}$, Yanda $\mathrm{Ji}^{3}$, Chang $\mathrm{Lu}^{1}$, Min Gao ${ }^{1}$, Hao Yang ${ }^{3}$, Chonglin Chen ${ }^{4}$, Feng Pan ${ }^{2}$ and Yuan Lin ${ }^{1}$
}

Selectively modulating the sublattices in 3D transition metal oxides via strains could tailor the electronic configurations with emerging anomalous properties, which provides new platforms for fundamental researches as well as designs of devices. Here, we report tailoring the oxygen octahedral sublattices in vanadium dioxide $\left(\mathrm{VO}_{2}\right)$ thin films by anisotropic in-plane strains, and the observation of in-plane orientation-dependent metal-insulator transition. Through multimodal characterizations based on highresolution X-ray diffraction, electrical transport measurements, and polarization-dependent X-ray absorption spectroscopy at different temperatures, we demonstrate that nonequal strains were successfully induced along $A$ and B oxygen octahedral chains in $\mathrm{VO}_{2}$ films via a special design of epitaxial growth on vicinal substrates. The $\mathrm{V} 3 d^{1}$ orbital configurations are modulated in the two oxygen octahedral chains, resulting in in-plane orientation-dependent metal-insulator transition behaviors such as reduced hysteresis width and anisotropic phase transition temperature. This work provides new fundamental insights on metal-insulator transitions, and more importantly, opens up new opportunities for material and device developments

npj Quantum Materials (2019)4:39; https://doi.org/10.1038/s41535-019-0176-x

\section{INTRODUCTION}

Exploring exotic physical properties in 3D transition metal oxide films by using epitaxial elastic strains is a popular way in materials science. $^{1,2}$ Strain, as a tool of modifying the crystal lattice of the oxide films, can simultaneously modify the interaction between charge, orbital and spin degrees of freedom, which are strongly related to the physical properties of materials. ${ }^{2-5}$ For some 3D transition metal oxides, sublattices with different spatial symmetries often coexist in their crystal lattices, such as in $\mathrm{Fe}_{3} \mathrm{O}_{4}$, $\mathrm{CoFe} \mathrm{O}_{4}$, Vanadium dioxide $\left(\mathrm{VO}_{2}\right)$, and some superconductor cuprates crystal lattices., ${ }^{3,6,7}$ These sublattices, including oxygen octahedral, oxygen tetrahedron, and oxygen dodecahedron, are fundamental functional unit cells of oxides. Although strain engineering techniques have been developed for decades, ${ }^{8}$ controllably manipulating the sublattices of the 3D transition metal oxides still needs deep and systematical investigations. Especially, knowledge on correlation between the spatial symmetries of the sublattice unit cells and the symmetries of the charge, orbital and spin degrees of freedom of the 3D electrons inhabited in them, can provide a knob to tailor the sublattice of the 3D transition metal oxides using strain and thus to explore novel physical properties for practical applications.

$\mathrm{VO}_{2}$, a classical strong-correlated oxide that has a sharp metal-insulator transition (MIT) near $341 \mathrm{~K}$, is a typical 3D transition metal oxide consisting of two types of sublattices, i.e., two types of edge-shared oxygen octahedral chains. ${ }^{2,3,9,10}$ The MIT properties of $\mathrm{VO}_{2}$ mainly originate from the $\mathrm{V}-\mathrm{V}$ dimers inhabited in these two distinguishable edge-shared oxygen octahedral sublattices, which are called A octahedral chain and B octahedral chain. The A octahedral chain and the $B$ octahedral chain are arranged alternatively with a relative $90^{\circ}$ rotation around $C_{R}$ axial, as seen in Fig. 1a, b (a simplify structure depicture can be accessed in Fig. S1). During the $\mathrm{MIT}, \mathrm{VO}_{2}$ transfers from a rutile structure into a monoclinic structure, meanwhile the $\mathrm{V}$ ions shift and form the $\mathrm{V}-\mathrm{V}$ dimers. ${ }^{2,3,9,10}$ More exact to say, there is a shifting component along the $\mathrm{VO}_{2}[001]_{\mathrm{R}}$ direction for both $\mathrm{V}$ ions of the $\mathrm{A}$ octahedral chain and the $B$ octahedral chain. Besides, they have different shifting components in the $\mathrm{VO}_{2}(001)_{R}$ lattice plane. As shown in Fig. $1 \mathrm{~b}, \mathrm{~V}$ ions of $\mathrm{A}$ octahedral chain shift along $\mathrm{VO}_{2}[1 \overline{1} 0]_{R}$ direction, while $V$ ions of $B$ octahedral chain shift along $\mathrm{VO}_{2}[110]_{R}$ direction. Obviously, the deformation along $\mathrm{VO}_{2}[110]_{R}$ and $[1 \overline{1} 0]_{R}$ directions would strongly impact the shifting components of $\mathrm{V}$ ions. Since the MIT properties of $\mathrm{VO}_{2}$ have been demonstrated very sensitive to the strains along the $\mathrm{V}-\mathrm{O}$ bond, ${ }^{3,10}$ it is believed that the strains along $\mathrm{VO}_{2}[110]_{R}$ and $[1 \overline{1} 0]_{R}$ directions would be one of the key factors affecting the MIT properties of $\mathrm{VO}_{2}$.

The strains along $\mathrm{VO}_{2}[110]_{R}$ and $[1 \overline{1} 0]_{R}$ directions can tailor the apical $\mathrm{V}-\mathrm{O}$ bond length (defined in Fig. 1c) of $\mathrm{VO}_{2}$, which influences the overlap of $\mathrm{V} 3 d$ and oxygen $2 p$ orbitals, hence changes the electronic configuration of $\mathrm{VO}_{2}$ and its MIT properties. $^{2,3}$ The $\mathrm{V}$ ions inhabit in the center of oxygen-octahedral cage and the crystal field of the oxygen octahedral splits the $3 d$ orbital into twofold degenerate $e_{g}$ orbitals and triple fold degenerate $t_{2 g}$ orbitals. The $e_{\mathrm{g}}$ orbitals contain $d_{\mathrm{x}^{2}+\mathrm{y}^{2}}$ and $d_{\mathrm{z}^{2}}$ which directly point toward the oxygen ions, hence lifting up their energy levels relative to the $t_{2 \mathrm{~g}}$ orbitals. In this case, the $\mathrm{V} d^{1}$ electron would occupy the $t_{2 \mathrm{~g}}$ orbital rather than the $e_{\mathrm{g}}$ orbital. ${ }^{5}$ For the $t_{2 \mathrm{~g}}$ orbitals $\left(d_{x y}, d_{x z}\right.$ and $\left.d_{y z}\right)$, the structural distortion of $\mathrm{VO}_{2}$ further splits the orbitals into $d_{\|}\left(d_{\mathrm{xy}}\right)$ and $\pi^{*}\left(d_{\mathrm{xz}}\right.$ and $\left.d_{\mathrm{yz}}\right){ }^{2,4}$ The $\mathrm{V} 3 d^{1}$ electron occupies $d_{\|}$orbital in the insulating state of $\mathrm{VO}_{2}$. However, in the metallic $\mathrm{VO}_{2}$, the electrons normally redistribute and occupy both the $d_{\|}$and $\pi^{*}$ orbitals (as shown in Fig. S2). The

\footnotetext{
${ }^{1}$ State Key Laboratory of Electronic Thin Films and Integrated Devices, University of Electronic Science and Technology of China, Chengdu, Sichuan 610054, PR China; ${ }^{2}$ School of

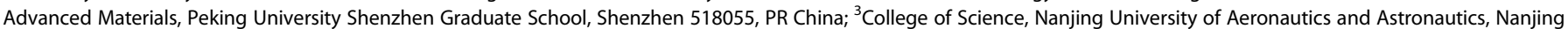
211106, PR China and ${ }^{4}$ Department of Physics and Astronomy, University of Texas at San Antonio, San Antonio, TX 78249, USA

Correspondence: Min Gao (mingao@uestc.edu.cn) or Yuan Lin (linyuan@uestc.edu.cn)
}

Received: 16 December 2018 Accepted: 26 June 2019

Published online: 26 July 2019 

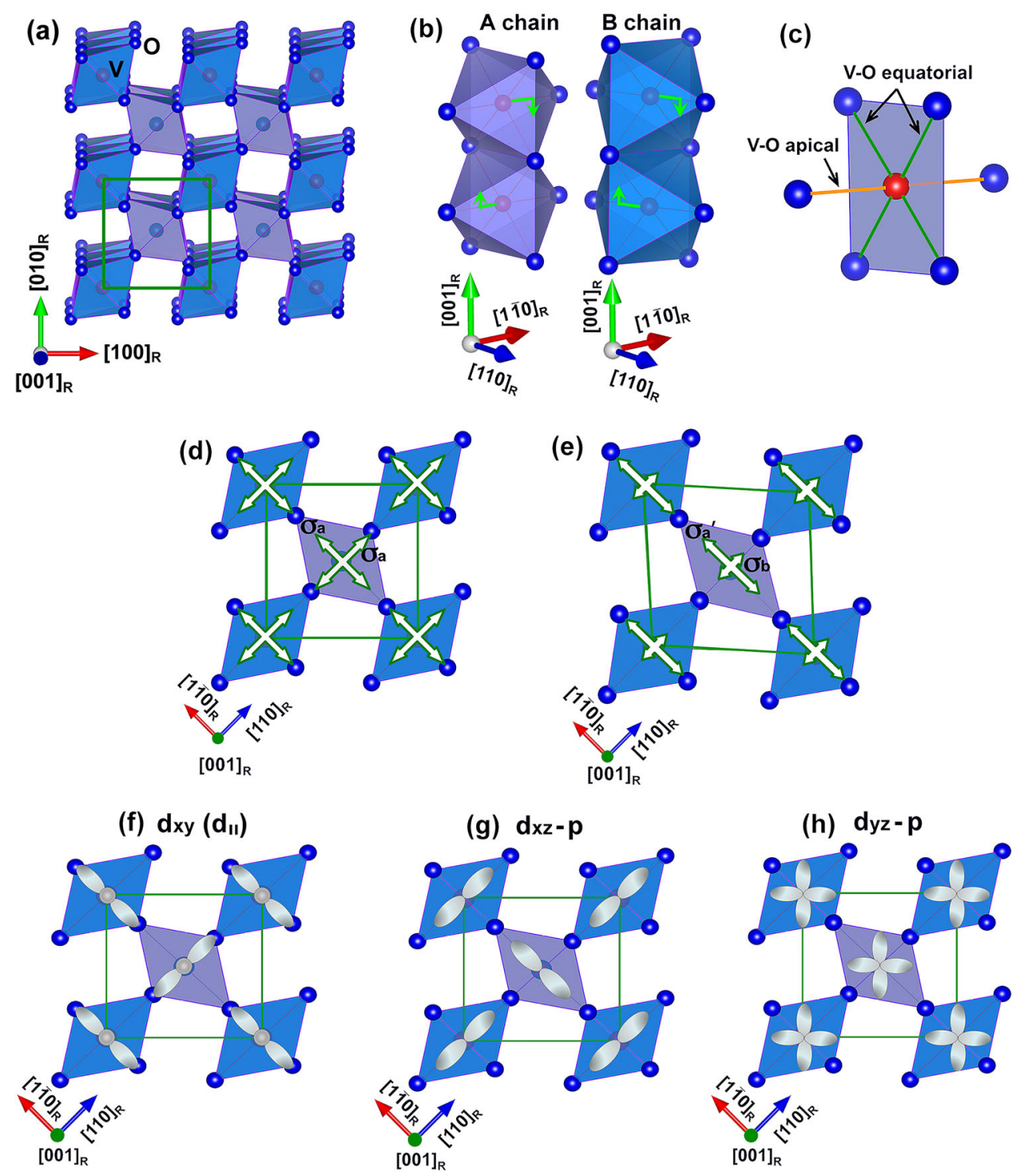

Fig. 1 The schematic of lattice and electronic structures for $\mathrm{VO}_{2}$ : a the $\mathrm{VO}_{2}$ lattice constructed with $\mathrm{A}$ and $\mathrm{B}$ oxygen octahedral chains which are edge-shared oxygen octahedral chains with $\mathrm{V}$ ions located inside the oxygen octahedral; $\mathbf{b}$ the details of $\mathrm{A}$ and $\mathrm{B}$ octahedral chains of $\mathrm{VO} \mathrm{O}_{2}$; c the definition of $\mathrm{V}-\mathrm{O}$ apical bonds and equatorial bonds of oxygen octahedral in $\mathrm{VO}_{2}$; d the lattice of $\mathrm{VO}_{2}$ grown on a normal TiO $(001)$ substrate, which undergoes symmetric biaxial strains along $\mathrm{VO}_{2}[110]_{R}$ and $[1 \overline{10}]_{R}$ directions; e the lattice of $\mathrm{VO}_{2}$ grown on a vicinal TiO $2(001)$ substrate with asymmetric biaxial strains along $\mathrm{VO}_{2}[110]_{R}$ and $[1 \overline{1} 0]_{R}$ directions, in which the $\mathrm{VO}_{2}[110]_{R}$ direction is the vicinal direction and bares a smaller strain compared with $\mathrm{VO}_{2}[1 \overline{1} 0]_{\mathrm{R}}$ direction; $(\mathbf{f}-\mathbf{h})$ the schematic of the $d_{\mathrm{xy}}, d_{\mathrm{xz}}$, and $d_{\mathrm{yz}}$ molecular orbitals of $\mathrm{VO}_{2}$ observed from $\mathrm{VO}_{2}[001]_{\mathrm{R}}$ direction, respectively

redistribution of $\mathrm{V} 3 \mathrm{D}$ electrons is very sensitive to the energy levels of $d_{\|}$and $\pi^{*}$ orbitals, which are strongly influenced by the apical $\mathrm{V}-\mathrm{O}$ bond length. ${ }^{3}$ If we observe along the $\mathrm{c}_{\mathrm{R}}$ direction, the $d_{x y}, d_{x z}$ and $d_{y z}$ orbitals extend to different directions in $\mathrm{VO}_{2}(001)_{R}$ plane (as shown in Fig. $1 \mathrm{f}-\mathrm{h}$ ). ${ }^{4,5}$ The $A$ octahedral chain and the $B$ octahedral chain have a relative $90^{\circ}$ rotation around $C_{R}$ axis, so their apical V-O bond and orbitals also rotate. Since the energy level of $\pi^{*}$ is sensitive to the apical $\mathrm{V}-\mathrm{O}$ bond length, the energy levels of $\pi^{*}$ of $3 d^{1}$ electrons in A octahedral chain and the B octahedral chain would be influenced by strains with a relative $90^{\circ}$ rotation, respectively. When isotropic biaxial strains are loaded along $\mathrm{VO}_{2}[110]_{R}$ and $[1 \overline{1} 0]_{R}$ directions(as the case of Fig. 1d), the apical $\mathrm{V}-\mathrm{O}$ bond lengths of both the $\mathrm{A}$ octahedral chain and the $\mathrm{B}$ octahedral chain would be tuned to the same degree simultaneously, as reported by Aetukuri et.al. ${ }^{3}$ The isotropic biaxial strains loaded along $\mathrm{VO}_{2}[110]_{R}$ and $[1 \overline{1} 0]_{R}$ directions can modulate the electronic configurations of $\mathrm{V} 3 \mathrm{D}$ electrons, consequently significantly tune the MIT temperature. Since the strains along $\mathrm{VO}_{2}[110]_{R}$ and $[1 \overline{1} 0]_{R}$ directions can selectively influence the apical V-O bond lengths of the A octahedral chain and the B octahedral chain, their sublattices could be tuned separately with nonequal strains along $\mathrm{VO}_{2}[110]_{R}$ and $[1 \overline{1} 0]_{R}$ directions. In other words, the orbital configurations of the A octahedral chain and the B octahedral chain can be tailored separately by the anisotropic strains(as the case of Fig. 1e). High-quality works on tailoring the MIT properties of $\mathrm{VO}_{2}$ in virtue of thickness effects on the epitaxial strain, ${ }^{11,12}$ thermal strain, ${ }^{13}$ and interface facet effect ${ }^{14}$ were reported. However, researches on the effect of anisotropic strains are still rare. Although the experiments of loading uniaxial strain along the $[110]_{R}$ direction have been demonstrated in $\mathrm{VO}_{2}$ nanowire system, ${ }^{10,15}$ the impact of the applied strain on the physical properties was hardly determined due to the dimension limitation. Furthermore, there is few data available on the anisotropic electron transport properties and the electronic 
configurations under the anisotropic strains. On the other hand, it has been demonstrated that substrates with small miscutting angles can induce designable strains in the films by virtue of the mismatching between the surface-step-terrace of the substrate and lattice unit cells of the films. ${ }^{16-19}$ For the substrates with miscutting angles, the surface-step-terrace can be formed after being annealed at a high temperature and its width can be adjusted by tuning the miscutting angles. When the films are grown on such substrates, the lattice unit cells of the films have to accommodate the width of surface-step-terrace, consequently strain is induced. (more details can be accessed in Supplementary Figs. S3 and S4). Herein, we induce nonequal strains in a $\mathrm{VO}_{2}$ film via high-quality epitaxial growth on a designed vicinal $\mathrm{TiO}_{2}(001)$ substrate with a small miscutting angle along $\mathrm{VO}_{2}[110]_{R}$ direction and directly observe an in-plane orientation-dependent MIT behavior of the as-grown $\mathrm{VO}_{2}$ film.

It is found that by high-quality epitaxial growth through sophisticated experimental control, anisotropic biaxial in-plane strains are induced in $A$ and $B$ oxygen octahedral chains in the $\mathrm{VO}_{2}$ film, resulting in an in-plane orientation-dependent MIT in $\mathrm{VO}_{2}$ film, e.g., obvious anisotropy in conductivity, crystal-orientation dependency of phase transition temperature $T_{c}$, and reduction of phase transition hysteresis width.

\section{RESULTS}

The $\mathrm{VO}_{2}$ films were grown using a polymer-assisted deposition technique. ${ }^{20}$ More details can be accessed in our previous reports. $^{21,22}$ The epitaxial relationship between the films and the substrates is $\mathrm{VO}_{2}(001)_{\mathrm{R}} \| \mathrm{TiO}_{2}(001)$ and $\mathrm{VO}_{2}[110]_{\mathrm{R}} \| \mathrm{TiO}_{2}[110]$ (Supplementary Fig. S5). And the thickness of the films is about $17 \mathrm{~nm}$. An ordinary $\mathrm{TiO}_{2}(001)$ substrate with no intentional miscut and a vicinal $\mathrm{TiO}_{2}(001)$ substrate with a $1^{\circ}$ miscutting angle along $\mathrm{TiO}_{2}$ [110] direction were used for comparative study. The anisotropic in-plane strains were successfully induced in the $\mathrm{VO}_{2}$ film on the vicinal $\mathrm{TiO}_{2}(001)$ substrate as manifested by the highresolution reciprocal space mapping (RSM) (Fig. 2). For the film grown on the normal $\mathrm{TiO}_{2}(001)$ substrate, equal strain of $\sigma_{\mathrm{a}}$ was found along both $\mathrm{VO}_{2}[110]_{R}$ and $[1 \overline{1} 0]_{R}$ directions in the $\mathrm{VO}_{2}$ film (Fig. 1d). However, the case was found to be different for the $\mathrm{VO}_{2}$ film grown on the vicinal $\mathrm{TiO}_{2}$ (001) substrate. We denoted the strain in the film grown on the vicinal substrate along the $\mathrm{VO}_{2}$ $[110]_{R}$ direction as $\sigma /_{\mathrm{a}}$ and along the $\mathrm{VO}_{2}[1 \overline{1} 0]_{\mathrm{R}}$ direction as $\sigma_{\mathrm{b}}$ (Fig. 1e). It was found that the strain $\sigma \sigma_{\mathrm{a}}$ is very close to $\sigma_{\mathrm{a}}$ but the strain $\sigma_{\mathrm{b}}$ is different from the strain $\sigma_{\mathrm{a}}$. Obviously, the strain along the $\mathrm{VO}_{2} \quad[110]_{\mathrm{R}}$ direction was modulated by the surface-step-terraces on the vicinal substrate, as what it can be expected.

Figure 2 show the RSMs for the nonvicinal and vicinal samples in both the metallic and insulating states, measured at 298 and $373 \mathrm{~K}$, respectively. Lattice parameters of the $\mathrm{VO}_{2}$ thin films are calculated based on the RSM data and listed in Table 1. It is clearly indicated that the anisotropic in-plane biaxial strains $\left(\sigma_{\mathrm{a}}^{\prime}\right.$ and $\left.\sigma_{\mathrm{b}}\right)$ have been induced along $[110]_{R}$ and $[1 \overline{1} 0]_{R}$ directions in the $\mathrm{VO}_{2}$ film on the vicinal substrate. The most profound influence of the anisotropic in-plane strains on the $\mathrm{VO}_{2}$ lattice is the ability of tailoring the apical $\mathrm{V}-\mathrm{O}$ bond length of the sublattices in the different octahedral chains separately. The A octahedral chain bares the $\sigma_{\mathrm{a}}{ }^{\prime}$ strain while the B octahedral chain bares the $\sigma_{\mathrm{b}}$ strain along the apical $\mathrm{V}-\mathrm{O}$ bond direction. The anisotropic in-plane biaxial strains will result in different apical V-O bond lengths in the $A$ and $B$ octahedral chains. From the measured lattice parameter in Table 1, we can estimate the apical V-O bond lengths for both samples in the metallic states. For the nonvicinal sample,
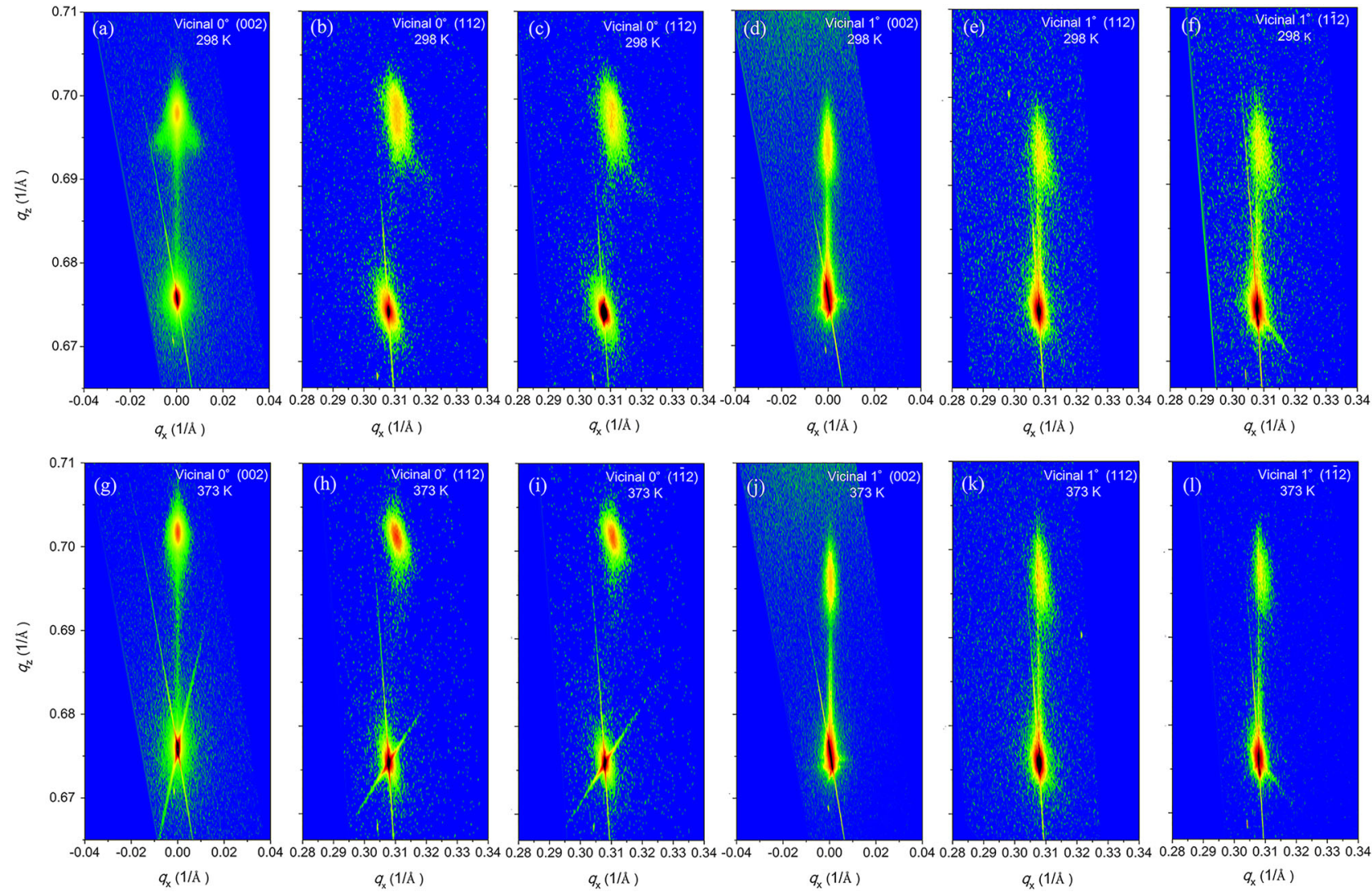

Fig. 2 The high-resolution reciprocal space maps (RSMs). (a-f) (002), (112) and (11/2) diffractions of the nonvicinal and vicinal samples at $298 \mathrm{~K}$, respectively. (g-I) (002), (112) and (1112) diffractions of the nonvicinal and vicinal samples at $373 \mathrm{~K}$, respectively 
W. Liang et al.

Table 1. Lattice parameters (Unit: $\AA$ ) and the corresponding strains rate (Unit: \%) of the $\mathrm{VO}_{2}$ thin films calculated based on the RSM data.(Unit: $\AA$ )

\begin{tabular}{lllrrrrr}
\hline Sample & Temperature & $d_{[002]}$ & $\sigma_{[002]}$ & $d_{[110]}$ & \multicolumn{1}{c}{$\sigma_{[110]}$} & $\mathbf{d}_{[1 \overline{1} 0]}$ \\
\hline Nonvicinal & $298 \mathrm{~K}$ & 1.4329 & 0.238 & 3.2285 & 0.202 & 3.2300 & $\boldsymbol{\sigma}_{[1 \overline{0} 0]}$ \\
Nonvicinal & $373 \mathrm{~K}$ & 1.4251 & -0.308 & 3.2234 & 0.043 & 3.2204 \\
Vicinal & $298 \mathrm{~K}$ & 1.4423 & 0.895 & 3.2187 & -0.102 & 3.2543 \\
Vicinal & $373 \mathrm{~K}$ & 1.4365 & 0.490 & 3.2013 & -0.642 & 3.2564 & 1.003 \\
\hline
\end{tabular}

obviously, the apical V-O bond lengths can be considered as the same for both A octahedral chain and B octahedral chain, which are determined to be $1.9331 \AA$ in the metallic state. However, for the vicinal sample, they become $1.9538 \AA$ and $1.9208 \AA$ for the $A$ octahedral chain and the $B$ octahedral chain in the metallic state, respectively. The nonequal apical $\mathrm{V}-\mathrm{O}$ bonds in the A octahedral chain and the $B$ octahedral chain for the vicinal sample are resulted from the nonequal biaxial strains.

The change of the apical $\mathrm{V}-\mathrm{O}$ bond length by strain would affect the overlap of $\mathrm{V} 3 d$ and $\mathrm{O} 2 p$ orbitals. A shorter apical $\mathrm{V}-\mathrm{O}$ bond length increases the overlap of $\mathrm{V} 3 d$ and $\mathrm{O} 2 p$ orbitals, which would increase the energy level of $\pi^{*}$ orbitals relative to those of the $d_{\|}$orbitals, hence decrease the electron occupancy of $\pi^{*}$ orbitals. Since the $d_{\|}$and $\pi^{*}$ orbitals are both partially occupied in the metallic state, as shown in Fig. S2, the electron occupancy of $\mathrm{d}_{\|}$orbitals will increase correspondingly. ${ }^{3}$ In other words, the nonequal strains in the $\mathrm{VO}_{2}$ film along $\mathrm{VO}_{2}[110]_{R}$ and $[1 \overline{1} 0]_{R}$ directions result in nonequal apical $\mathrm{V}-\mathrm{O}$ bond lengths in $\mathrm{A}$ and $\mathrm{B}$ octahedral chains, leading to different energy levels of $\pi^{*}$ orbitals and different electronic configurations of the $A$ and $B$ octahedral chains.

To verify the electronic state configurations described above, synchrotron-based linear-polarization-dependent X-ray absorption spectroscopy (XAS) was tested at the $\mathrm{VL}_{2,3}$ edges of both $\mathrm{VO}_{2}$ thin films epitaxially grown on non-vicinal and vicinal substrates. The experiments were performed using total electron yield (TEY) detection with a typical probe depth of about $10 \mathrm{~nm}$. Linearly polarized X-rays with the electric-field orientation parallel $\left(E \perp c_{R}\right)$ and perpendicular $\left(E \perp C_{R}\right)$ to the rutile $\mathrm{VO}_{2} c$-axis are measured to probe the vacant $d_{\|}$and $\pi^{*}$ valence-electron states, respectively. ${ }^{3}$ To investigate the anisotropic in-plane strain effect, the measurements perpendicular to the rutile $c$-axis $\left(E \perp C_{R}\right)$ were performed along $\mathrm{VO}_{2}[110]_{R}$ and $[1 \overline{1} 0]_{R}$ directions, respectively. Based on previous studies on the valence-electron states, ${ }^{3}$ we particularly focus on the excitonic features of the XAS spectra with 512-516 eV photon energies.

Figure 3 shows the polarization-dependent $V$ L-edge XAS spectra in the metallic and insulating states of the nonvicinal and vicinal samples. The intensity differences $I_{\|}-I_{\perp}$, which are respectively defined as $I_{C_{R}}-I_{[110]}$ and $I_{C_{R}}-I_{[110]}$ along the $\mathrm{VO}_{2}$ $[110]_{R}$ and $[1 \overline{1} 0]_{R}$ directions, are also plotted. In the insulating state, the dichroic signals $I_{C_{R}}-I_{[110]}$ and $I_{C_{R}}-I_{[1 \overline{1} 0]}$ (Fig. 3a, b) of both nonvicinal and vicinal samples show negligible difference. That is expected and consistent with the previous report, ${ }^{3}$ because the $3 d^{1}$ electron occupies the $d_{\|}$orbitals while the $\pi^{*}$ orbitals located above the Fermi level are empty in the insulating state. However, the orbital occupancy in the metallic state, which is believed to set the energy scale for the MIT of $\mathrm{VO}_{2}{ }^{3}$ shows remarkable distinctions between the two samples, as reflected by the XAS data. It is found that the XAS dichroic signals, $I_{C_{R}}-I_{[110]}$ and $I_{C_{R}}-I_{[1 \overline{1} 0]}$, of the nonvicinal sample in the energy region of 512-516 eV show negligible difference in the metallic state (Fig. 3c), which is as expected due to the equal strains along $\mathrm{VO}_{2}[110]_{R}$ and $[1 \overline{1} 0]_{R}$ directions. On the other hand, the XAS dichroic signals $I_{C_{R}}-I_{[110]}$ and $I_{C_{R}}-I_{[1 \overline{1} 0]}$ of the vicinal sample in the metallic state (Fig. 3d), show obvious difference in this energy region, which should be attributed to the nonequal strains along $\mathrm{VO}_{2}[110]_{R}$ and $[1 \overline{1} 0]_{R}$ directions. For the vicinal sample, the apical $\mathrm{V}-\mathrm{O}$ bond length of the $B$ octahedral chain (1.9208 $\AA$ ) was shorter than the $A$ octahedral chain $(1.9538 \AA)$, suggesting that the difference in the XAS dichroic signals $I_{C_{R}}-I_{[110]}$ and $I_{C_{R}}-I_{[110]}$ is due to the redistribution of the orbital occupation. As mentioned ahead, the shorter apical $\mathrm{V}-\mathrm{O}$ bond length increases the $p-d$ overlap, hence raises the energy level of $\pi^{*}$ orbital and consequently reduces their orbital occupancy, resulting in the increase of the orbital occupancy of $d_{\|}$orbitals. Since the XAS data correspond to the unoccupied states of the orbitals, the increase of the occupancy of $\mathrm{d}_{\|}$orbitals will reduce the signal $l_{C_{R}}$. For the $\pi^{*}$ orbitals, the $d_{\mathrm{yz}}$ orbitals extend to $[100]_{R}$ and $[010]_{R}$ directions (Fig. 1h). Thus, the related XAS signals display no difference with the $\mathrm{X}$-ray electric field along either $[110]_{R}$ or $[1 \overline{1} 0]_{R}$ directions. However, the $d_{x z}$ orbitals of $B$ octahedral chain extend along $[110]_{R}$ direction (Fig. 1g) while the $d_{x z}$ orbitals of A octahedral chain extend along $[1 \overline{1} 0]_{R}$ direction. The anisotropic in-plane strain effect, i.e., a shorter lattice parameter along the $[110]_{R}$, would increase the energy level and decrease the orbital occupancy of the $d_{x z}$ orbitals in the B octahedral chain, compared with those in the A octahedral chain. Such an effect is directly shown in the XAS raw data plot in Fig. $3 d$, with stronger $I_{[110]_{R}}$ signals comparing with $I_{[1 \overline{1} 0]_{R^{\prime}}}$, leading to smaller $I_{C_{R}}-I_{[110]}$ than $I_{C_{R}}-I_{[1 \overline{1} 0]}$. Therefore, the XAS data in the metallic states clearly prove that the anisotropic in-plane strains have effectively changed the orbital occupancy of the two sublattices.

Since the nonequal apical $\mathrm{V}-\mathrm{O}$ bonds of the A octahedral chain and the $\mathrm{B}$ octahedral chain change the electronic configurations of the $\mathrm{VO}_{2}$ film on the vicinal substrate, it can significantly modulate the MIT behaviors of the film. Temperature-dependent resistances of both the nonvicinal and the vicinal samples were tested. In-plane orientation-dependent MIT behavior attributed to the anisotropic inplane strains were observed in the vicinal sample.

The nonvicinal sample exhibits a normal MIT behavior (Fig. 4a, c). The resistance-temperature $(R-T)$ curves tested along $\mathrm{VO}_{2}[110]_{R}$ and $[1 \overline{1} 0]_{R}$ directions show weak directional dependency, as shown in the direction-dependent resistance in metallic state is shown in Fig. 4e. Its phase transition temperature $\left(T_{c}\right)$ for heating was determined to be $324 \mathrm{~K}$ in both $\mathrm{VO}_{2}[110]_{\mathrm{R}}$ and $[1 \overline{10}]_{\mathrm{R}}$ directions (derived from the $\mathrm{d} \ln R / \mathrm{d} T-T$ curves in Fig. $4 \mathrm{c}$ ), and the hysteresis width ( $\Delta T_{c}$, defined as the difference of $T_{c}$ for heating and cooling) is about $3.5 \mathrm{~K}$. These results are very similar to previous reports. ${ }^{23,24}$ However, for the vicinal sample, the MIT behavior has been obviously altered (Fig. 4b, d).The $R-T$ curves tested along $\mathrm{VO}_{2}$ $[110]_{R}$ and $[1 \overline{1} 0]_{R}$ directions show obvious directional dependency. The film in the metallic state has a much larger resistance along the $\mathrm{VO}_{2}[110]_{\mathrm{R}}$ direction (vicinal direction) than that along the $\mathrm{VO}_{2}$ $[1 \overline{10}]_{R}$ direction. The direction-dependent resistance in metallic state is shown in Fig. $4 \mathrm{f}$, which can be fitted by:

$R(\theta)=R_{[110]_{R}} \sin ^{2}(\theta)+R_{[1 \overline{1} 0]_{R}} \cos ^{2}(\theta)$

where $R_{[110]_{R}} / R_{[1 \overline{1} 0]_{R}}=17.3$. The phase transition temperature exhibits anisotropy as well. The $T_{\mathrm{c}}$ values measured along $[110]_{\mathrm{R}}$ and $[1 \overline{1} 0]_{R}$ directions are determined to be 333 and $326 \mathrm{~K}$, respectively. From Table 1 , we observe that the vicinal sample 

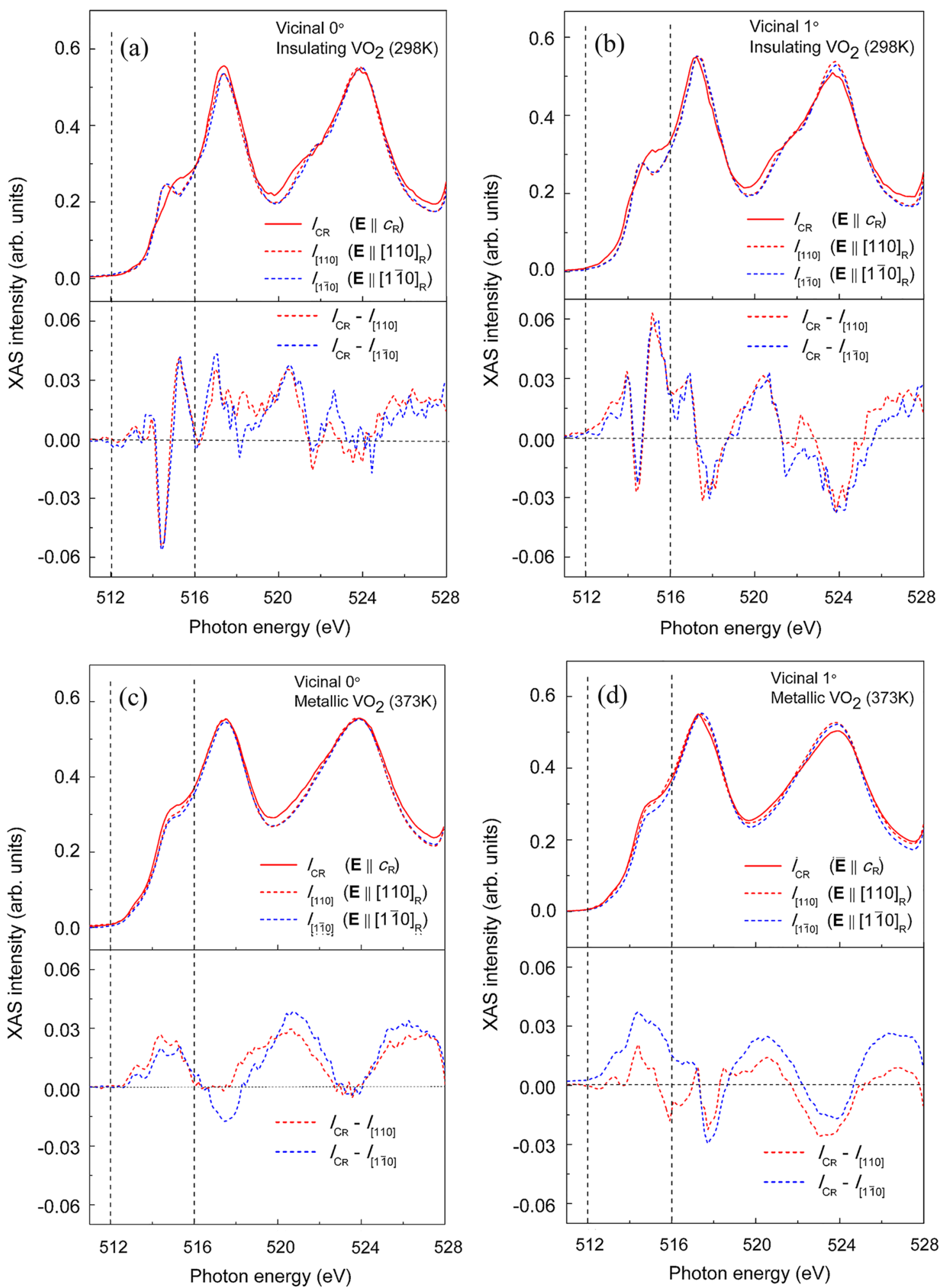

Fig. 3 The polarization-dependent V L-edge XAS spectra. $\mathbf{a}$, b Polarization-dependent V L-edge XAS spectra in the insulating state of the nonvicinal and vicinal samples, respectively; $\mathbf{c}$, $\mathbf{d}$ polarization-dependent $\mathrm{V}$ L-edge XAS spectra in the metallic state of the nonvicinal and vicinal samples, respectively

undergoes a tensile strain along the $[1 \overline{1} 0]_{R}$ direction, while it undergoes a compressive strain along the $[110]_{R}$ direction. The higher value of $T_{\mathrm{c}}$ along the $[110]_{\mathrm{R}}$ direction should be attributed to the compressive strain along that direction.

\section{DISCUSSION}

A possible mechanism for the conductivity anisotropy is the striplike phase domain structure generated by the phase separation during the phase transition in the film. ${ }^{25}$ Clearly, mixture of the 

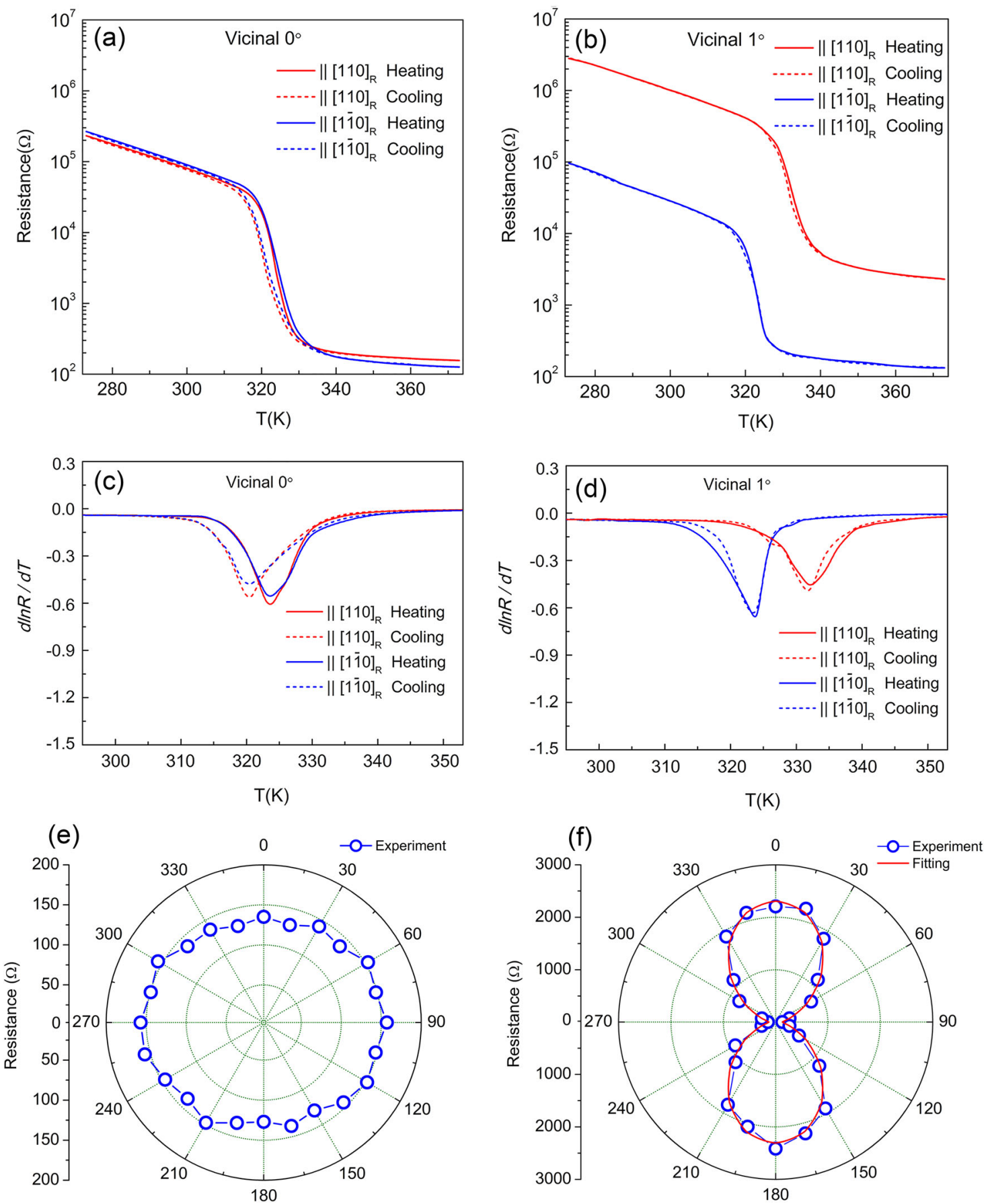

Fig. 4 The electrical properties. a Temperature-dependent resistance and c $\operatorname{dln} R / \mathrm{d} T-T$ curves of the nonvicinal sample, respectively; b temperature-dependent resistance and $\mathbf{d} \mathrm{d} \ln R / \mathrm{d} T-T$ curves of the vicinal sample, respectively; $\mathbf{e}$, $\mathbf{f}$ the experimental and fitting results of direction-dependent resistance for the nonvicinal and vicinal sample in metallic state, respectively

metallic and insulating phases would occur in the temperature range near the phase transition temperature. This mixing phase domains would form anisotropic percolation hence lead to anisotropic carrier transport behavior. Anisotropy of the conductivity induced by this mechanism will disappear beyond the phase transition temperature range, since the phase transition has completed and the metallic and insulating domains no longer coexist. However, our vicinal sample shows obvious anisotropic conductivity even at a high ( $373 \mathrm{~K}$ ) and a low (298 K) temperature beyond the phase transition temperature. Other mechanisms need to be proposed to understand the phenomenon. We suggest that the anisotropic in-plane strains play a key role. The shorter apical V-O bond in the B octahedral chain compared with the A octahedral chain increases the $p-d$ overlap and the $d_{x z}$ energy level. Since the $d_{\mathrm{yz}}$ orbitals of the $\mathrm{B}$ octahedral chain extend to $[110]_{R}$ direction while those of the $A$ octahedral chain extend to $[1 \overline{10}]_{R}$ direction, if we assume that the $d_{\|}$orbital levels are the same in the $A$ and $B$ octahedral chains, the electron hopping would be harder along $[110]_{R}$ than $[1 \overline{1} 0]_{R}$. As a result, the resistance along $[110]_{R}$ is larger than that along $[1 \overline{1} 0]_{R}$.

Moreover, the nonequal apical $\mathrm{V}-\mathrm{O}$ bonds change the electronic configurations of the $A$ octahedral chain and the $B$ 
octahedral chain, leading to different energy scales for the MIT along $[1 \overline{1} 0]_{R}$ and $[110]_{R}$ directions. Since the apical $V-O$ bond length expands when $\mathrm{VO}_{2}$ transfers from a low temperature monoclinic phase to a rutile phase, a longer apical $\mathrm{V}-\mathrm{O}$ bond length would make the phase transition easier, therefore yielding a lower transition $T_{c}$ along $[1 \overline{10}]_{R}$ than $[110]_{R}$.

An anomalous change in the phase transition hysteresis width was also observed in the vicinal sample. As shown in Fig. 4, the hysteresis width $\Delta T_{\mathrm{c}}$ almost disappeared in the vicinal sample, i.e, the values of $\Delta T_{\mathrm{c}}$ are as small as $0.2 \mathrm{~K}$ along both $\mathrm{VO}_{2}[110]_{\mathrm{R}}$ and $[1 \overline{10}]_{R}$ directions, which are much smaller than that of the nonvicinal sample $\left(\Delta T_{\mathrm{c}}=3.5 \mathrm{~K}\right)$. The reason for the dramatically reduced hysteresis width $\Delta T_{\mathrm{C}}$ is not clear yet. It may be related to the modification of the shearing strain in the $\mathrm{VO}_{2}$ film during the MIT. In the previous reports, ${ }^{26,27}$ the hysteresis width can be expressed as:

$\Delta T_{\mathrm{c}}=T_{\text {heating }}-T_{\text {cooling }}=\frac{\eta G \gamma^{2}}{2 \Delta S}$,

where $\eta$ is the domain shape parameter, $G$ is the shear modulus, $\gamma$ is the shearing strain, and $\Delta S$ is entropy change of the phase transition. The asymmetrical in-plane strains in the film may alter the shearing strain $\gamma$ hence narrow the hysteresis loop.

By epitaxial growth of a high-quality $\mathrm{VO}_{2}$ thin film on a designed vicinal $\mathrm{TiO}_{2}$ substrate, we deliberately tailored the sublattices of $\mathrm{VO}_{2}$ crystal utilizing the nonequal biaxial in-plane strains. The anisotropic in-plane strain leads to different apical V-O bond lengths in $\mathrm{A}$ and $\mathrm{B}$ octahedral chains of the $\mathrm{VO}_{2}$, which modulates the orbital configurations of $\mathrm{V} 3 d^{1}$ and induces in-plane orientation-dependent MIT behaviors in the $\mathrm{VO}_{2}$ film. Specifically, direction-dependent conductivity and phase transition temperature $T_{\mathrm{c}}$, as well as the significant reduction of the hysteresis width $\Delta T_{c}$, have been observed. Our experimental results demonstrate the feasibility of tailoring the sublattices in the 3D transition metal oxides for modulating their electronic configure using anisotropic strains. This demonstration presents a unique platform in the material genetic design for exploring both fundamental physical properties and practical applications.

\section{METHODS}

The vicinal $\mathrm{TiO}_{2}(001)$ substrate and the normal $\mathrm{TiO}_{2}(001)$ substrate were both ultrasonically cleaned in acetone and deionized water, then immersed in 5 vol\% $\mathrm{HCl}$ solution for $5 \mathrm{~min}$ to remove the metal contamination on the surfaces of the substrates before being washed in deionized water. After that, the substrates were etched in $20 \mathrm{vol} \% \mathrm{HF}$ solution for $2 \mathrm{~min}$, and then ultrasonically cleaned in deionized water. Finally, the substrates were annealed in oxygen at $750{ }^{\circ} \mathrm{C}$ for $2 \mathrm{~h}$ to form a surface with clear step-terrace structures. The high-resolution reciprocal space maps were tested using PANalytical XPert MRD. The temperature dependence of resistance of the films was measured using an Agilent B2900A source meter with the four-point probes method in a high-vacuum system. The anisotropic resistance of the $\mathrm{VO}_{2}$ films was also measured with the four-point probes method by rotating the samples. The temperaturedependent polarization-dependent XAS was carried out with $>99 \%$ linearly polarized X-ray beam, which was carefully aligned onto the samples mounted on an in-vacuum rotating goniometer with temperature controls. Sample current was collected as the TEY signals upon incident photons. The XAS spectra shown here have been normalized to the photon flux that was monitored by the photocurrent of a clean gold mesh simultaneously. Experimental resolution is better than $0.16 \mathrm{eV}$ at $\mathrm{V}-\mathrm{L}$ edges, not considering core-hole broadening.

\section{DATA AVAILABILITY}

All data supporting the findings of this study are available from the corresponding authors Y.L. and M.G. upon request.

\section{ACKNOWLEDGEMENTS}

This work has been supported by the National Basic Research Program of China (973 Program) under Grant no. 2015CB351905 and the Natural Science Foundation of China (Nos. 61825102, 51872038 and 51602152).

\section{AUTHOR CONTRIBUTIONS}

Y.L., C.C. and W.L. discussed and initiated the study and Y.L. supervised the project. W. L. conducted the fabrication experiments. Z.Z. conducted the XAS measurements. Y.J. and H.Y. performed the RSM measurements. C.L. and M.G. performed the electric measurement. W.L., Z.Z., Y.J., H.Y., M.G., C.C., F.P., and Y.L. analyzed the data and cowrote the manuscript. All authors reviewed and finalized the manuscript.

\section{ADDITIONAL INFORMATION}

Supplementary Information accompanies the paper on the npj Quantum Materials website (https://doi.org/10.1038/s41535-019-0176-x).

Competing interests: The authors declare no competing interests.

Publisher's note: Springer Nature remains neutral with regard to jurisdictional claims in published maps and institutional affiliations.

\section{REFERENCES}

1. Schlom, D. G. et al. Elastic strain engineering of ferroic oxides. MRS Bull. 39 118-130 (2014).

2. Aetukuri, N. B. et al. Control of the metal-insulator transition in vanadium dioxide by modifying orbital occupancy. Nat. Phys. 9, 661-666 (2013).

3. Eyert, V. The metal-insulator transitions of $\mathrm{VO}_{2}$ : a band theoretical approach. Ann. Phys. 11, 650-704 (2010).

4. Morrison, V. R. et al. A photoinduced metal-like phase of monoclinic $\mathrm{VO}_{2}$ revealed by ultrafast electron diffraction. Science 346, 445-448 (2014).

5. Haverkort, M. W. et al. Orbital-assisted metal-insulator transition in $\mathrm{VO}_{2}$. Phys. Rev. Lett. 95, 196404 (2005).

6. Mcqueeney, R. J. et al. Zener double exchange from local valence fluctuations in magnetite. Phys. Rev. Lett. 99, 246401 (2007).

7. Fritsch, D. \& Ederer, C. First-principles calculation of magnetoelastic coefficients and magnetostriction in the spinel ferrites $\mathrm{CoFe}_{2} \mathrm{O}_{4}$ and $\mathrm{NiFe}_{2} \mathrm{O}_{4}$. Phys. Rev. B 86, 41-48 (2012)

8. Lin, Y. \& Chen, C. L. Interface effects on highly epitaxial ferroelectric thin films. J. Mater. Sci. 44, 5274-5287 (2009).

9. Goodenough, J. B. The two components of the crystallographic transition in $\mathrm{VO}_{2}$ J. Solid State Chem. 3, 490-500 (2015).

10. Park, J. H. et al. Measurement of a solid-state triple point at the metal-insulator transition in $\mathrm{VO}_{2}$. Nature 500, 431-434 (2013).

11. Théry, V., Boulle, A., Crunteanu, A. \& Orlianges, J. Combined strain and composition-induced effects in the metal-insulator transition of epitaxial $\mathrm{VO}_{2}$ films. Appl. Phys. Lett. 111, 251902 (2017).

12. Yang, Y. et al. Thickness effects on the epitaxial strain states and phase transformations in (001) $-\mathrm{VO}_{2} / \mathrm{TiO}_{2}$ thin films. J. Appl. Phys. 125, 082508 (2019).

13. Théry, V. et al. Role of thermal strain in the metal-insulator and structural phase transition of epitaxial $\mathrm{VO}_{2}$ films. Phys. Rev. B 93, 184106 (2016).

14. Corder, S. N. G. et al. Controlling phase separation in vanadium dioxide thin films via substrate engineering. Phys. Rev. B 96, 161110 (2017).

15. Wei, J., Wang, Z., Chen, W. \& Cobden, D. New aspects of the metal-insulator transition in vanadium dioxide nanobeams. Nat. Nanotechnol. 4, 420-424 (2009).

16. Sánchez, F., Ocal, C. \& Fontcuberta, J. Tailored surfaces of perovskite oxide substrates for conducted growth of thin films. Chem. Soc. Rev. 43, 2272-2285 (2014).

17. Perna, P. et al. Engineering large anisotropic magnetoresistance in $\mathrm{La}_{0.7} \mathrm{Sr}_{0.3} \mathrm{MnO}_{3}$ films at room temperature. Adv. Funct. Mater. 27, 1700664 (2017)

18. Lu, H. et al. Surface-step-terrace-induced anomalous transport properties in highly epitaxial $\mathrm{La}_{0.67} \mathrm{Ca}_{0.33} \mathrm{MnO}_{3}$ thin films. ACS Appl. Mater. Interfaces 2 2496-2499 (2010).

19. Zou, Q. et al. Step terrace tuned anisotropic transport properties of highly epitaxial $\mathrm{LaBaCO}_{2} \mathrm{O}_{5.5+\delta}$ thin films on vicinal $\mathrm{SrTiO}_{3}$ substrates. ACS Appl. Mater. Interfaces 6, 6704-6708 (2014).

20. Jia, Q. X. et al. Polymer-assisted deposition of metal-oxide films. Nat. Mater. 3, 529-532 (2004).

21. Ji, Y. D. et al. Epitaxial growth and metal-insulator transition of vanadium oxide thin films with controllable phases. Appl. Phys. Lett. 101, 071902 (2012).

22. Ji, $Y$. et al. Role of microstructures on the $m_{1}-m_{2}$ phase transition in epitaxial $V_{2}$ thin films. Sci. Rep. 4, 4854 (2014). 
23. Bayati, M. R. et al. Correlation between structure and semiconductor-to-metal transition characteristics of $\mathrm{VO}_{2} / \mathrm{TiO}_{2} /$ sapphire thin film heterostructures. Acta Mater. 61, 7805-7815 (2013).

24. Li, D. et al. Joule heating-induced metal-insulator transition in epitaxial $\mathrm{VO}_{2} / \mathrm{TiO}_{2}$ devices. ACS Appl. Mater. Interfaces 8, 12908-12914 (2016).

25. Liu, M. K. et al. Anisotropic electronic state via spontaneous phase separation in strained vanadium dioxide films. Phys. Rev. Lett. 111, 096602 (2013).

26. Thompson, Z. J. et al. Terahertz-triggered phase transition and hysteresis narrowing in a nanoantenna patterned vanadium dioxide film. Nano Lett. 15, 5893-5898 (2015).

27. Gu, Y., Cao, J., Wu, J. \& Chen, L. Q. Thermodynamics of strained vanadium dioxide single crystals. J. Appl. Phys. 108, 083517 (2010).
Open Access This article is licensed under a Creative Commons Attribution 4.0 International License, which permits use, sharing, adaptation, distribution and reproduction in any medium or format, as long as you give appropriate credit to the original author(s) and the source, provide a link to the Creative Commons license, and indicate if changes were made. The images or other third party material in this article are included in the article's Creative Commons license, unless indicated otherwise in a credit line to the material. If material is not included in the article's Creative Commons license and your intended use is not permitted by statutory regulation or exceeds the permitted use, you will need to obtain permission directly from the copyright holder. To view a copy of this license, visit http://creativecommons.org/licenses/by/4.0/.

(c) The Author(s) 2019 